\title{
Likert Data
}

\section{Samuel J. Stratton, MD, MPH}

Likert-scale and Likert-like data are frequently used in disaster research. Often, peer reviewers will challenge the statistical methods used for analysis of Likert data. While seemingly esoteric, Likert data management and analysis are worthy of discussion because many papers that are submitted to Prehospital and Disaster Medicine include this type of data. An interesting element for a seemingly simple data collection method is the lack of agreement among statisticians and researchers on how best to manage and analyze Likert data. ${ }^{1}$

The common form of Likert scale is an option of five to ten points measuring lack of support to strong support for the focus to a research question. The Likert-scale concept was developed in 1932 by Rensis Likert, ${ }^{2}$ with the intent to measure attitudes and beliefs of a study population. A frequent use of Likert-scale-based research is in satisfaction surveys that are frequently conducted in business product research. A common intent of researchers when using Likert-like data is to quantify "fuzzy" attitude data so that standard statistical methods can be used. In a simple definition, attitude is a way of thinking or feeling for an individual or group of individuals.

Proper use for a Likert-like response to a question is to determine the degree to which a respondent agrees or disagrees with the research question which is presented as a statement. A long-time statistical debate for Likert-like data centers around whether the data generated are ordinal or interval in character, as described by the Steven's Scale of Measurement. ${ }^{3}$ The original Likert system was based on an ordinal data scale in which responses are rated or ranked based on subjective (a feeling for) magnitude with the distance between numbered responses not measurable or mathematically meaningful. In contrast, interval (continuous) data have a difference between responses that can be calculated with the scale numbers measuring something mathematically meaningful. An example of interval data would be the number of rescued persons per hour, where " 5 " indicates an objective, measurable rate, or number.

The prime controversy with Likert data is whether it is appropriate to treat ordinal data (when this type is collected) as interval data. That is, can ordinal data generated by a Likert style question be analyzed using means (averages), standard deviations, and parametric statistics which rely on a standard bell-shape distribution and mathematically meaningful data? While some argue that application of mean and standard deviation statistical measures is appropriate for Likert data, most agree that ordinal data are appropriately described by mode, median, and quartiles. The common argument against applying parametric statistics (mean) to Likert data is that it is meaningless to measure a "strongly agree" response and an "agree" response within a set of Likert five-point responses and come up with a mathematically meaningful measure of "agree and $1 / 5$." This said, there are persuasive reviews of simulations and real data that show use of parametric tests such as means yield answers for Likert ordinal data that are unbiased and acceptable. ${ }^{1}$

In a more sophisticated approach to analysis of Likert data, many researchers will develop several Likert-like questions that are interrelated and address as a group of questions (survey scale) a particular outcome. In this use of Likert data, a single question is no longer the focus, rather usually three to five questions are developed to probe the outcome of interest. The questions are shown to be interrelated using the Cornbach alpha, Kappa test, or factor analysis. The total score for the interrelated group of questions is then used to calculate a mean score for the scale items that address the single topic of interest. ${ }^{4}$

A Likert data problem which is seldom addressed is the inherent bias of a population when researching attitudes. Likert data are designed for assessment of the attitudes of a population. If a research question can be framed to reliably provide nonattitude nominal or interval (continuous) data, a Likert data question is less preferred. The attitude of individuals and populations is at risk for strong bias due to exposure to media reports and discussion among family or interest groups regarding an event or issue. In addition, a person answering a Likert-style question is likely to express personality traits such as optimistic, negative, or disinterest attitude regarding the research topic. In considering these aspects of population attitude, it is important to realize Likert-measured attitude outcome may change without explanation due to external and internal biases and stresses for individuals.

Equally important as individual bias is careful selection of the study population for which Likert questions are being asked. Attitudes of specific groups will predictably vary. For example, asking hospital administrators if a hospital community is prepared for a certain disaster event will likely yield different answers (attitude) from the replies that would be received from daily patient care staff. Selection of those surveyed with Likert questions will determine the population for which the outcome conclusions apply. For example, unless a random study group including all of a hospital community is selected for survey, one cannot make valid conclusions regarding the whole of that hospital community.

In summary, use of Likert-type data is common in disaster research. Likert data are used to evaluate attitudes. Unless designed as interval data elements, Likert data are generally ordinal in nature and individual Likert questions are best analyzed using modes, medians, and quartiles. A considerable body of statistical literature suggests that application of parametric data measures such as means and standard deviation is valid for most Likert data. Parametric tests are more valid when using a survey scale method in which multiple interrelated Likert questions are evaluated to generate a mean measure of a single topic.

It is recommended when research is based on Likert data that how the questions are developed to address potential bias and ambiguity be a first action in organizing the research project. In designing Likert questions, it is important to determine how the data will be analyzed. When submitting a manuscript in which Likert data are analyzed, it is important to justify the data analysis 
used for making research conclusions. Finally, many authors will submit both an analysis using means and using medians to allow

References

1. Norman G. Likert scales, levels of measurement, and the "laws" of statistics. Adv Health Sci Educ Theory Pract. 2010;15(5):625-632.

2. Likert R. A technique for the measurement of attitudes. Arch Psychology. 1932;22 (140):55.

3. Stevens SS. On the theory of scales of measurement. Science. 1946;103(2684):677-680. readers and other researchers to see the data analysis from both sides of the "parametric wall."

4. Boone HN, Boone DA. Analyzing Likert data. JOE. 2012;50(2).

doi:10.1017/S1049023X18000237 